\title{
Facile Assembly of Multifunctional Antibacterial Nanoplatform Leveraging Synergistic Sensitization between Silver Nanostructure and Vancomycin
}

Kang Ma, ${ }^{+}$Pei Dong, ${ }^{+}$Meijuan Liang, Shanshan Yu, Yingying Chen, Fuan Wang*

Key Laboratory of Analytical Chemistry for Biology and Medicine (Ministry of

Education), College of Chemistry and Molecular Sciences, Wuhan University, Wuhan 430072, P. R. China.

* To whom correspondence should be addressed. E-mail: fuanwang@whu.edu.cn

† These authors contributed equally to this work. 


\section{Table of Contents}

Figure S1. Dynamic light scattering of different nanoparticles ............................ S-2

Figure S2. Characterization of PDA-Ag with increasing silver............................. S-3

Figure S3. Polydispersity index of different PDA-Ag nanoparticles....................... S-4

Figure S4. Representative TEM images of PDA@Van-Ag.................................... S-5

Figure S5. Loading and releasing of vancomycin ........................................... S-6

Figure S6. Photothermal PDA@Van-Ag nanohybrid ........................................... S-7

Figure S7. Photothermal bacteria elimination................................................. S-8

Figure S8. Ciprofloxacin against S.aureus and E.coli ....................................... S-9

Figure S9. Combinational therapy on E.coli ................................................ S-10

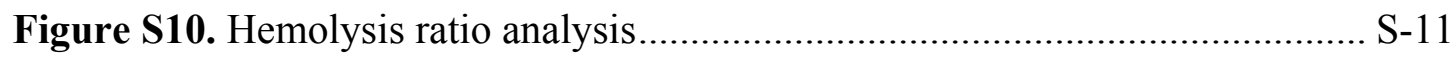

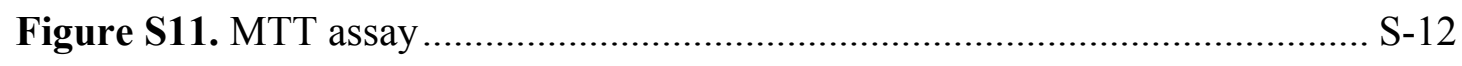

Figure S12. Blood and biochemical analysis .................................................. S-14

Table S1. In vivo silver distribution in major organs .......................................... S-13 
The size distribution of PDA and the ultimate PDA@Van-Ag nanohybrid was obtained from dynamic light scattering results (Figure S1). It revealed that the size of PDA@Van-Ag increased to some extent due to the successful deposition of AgNPs.
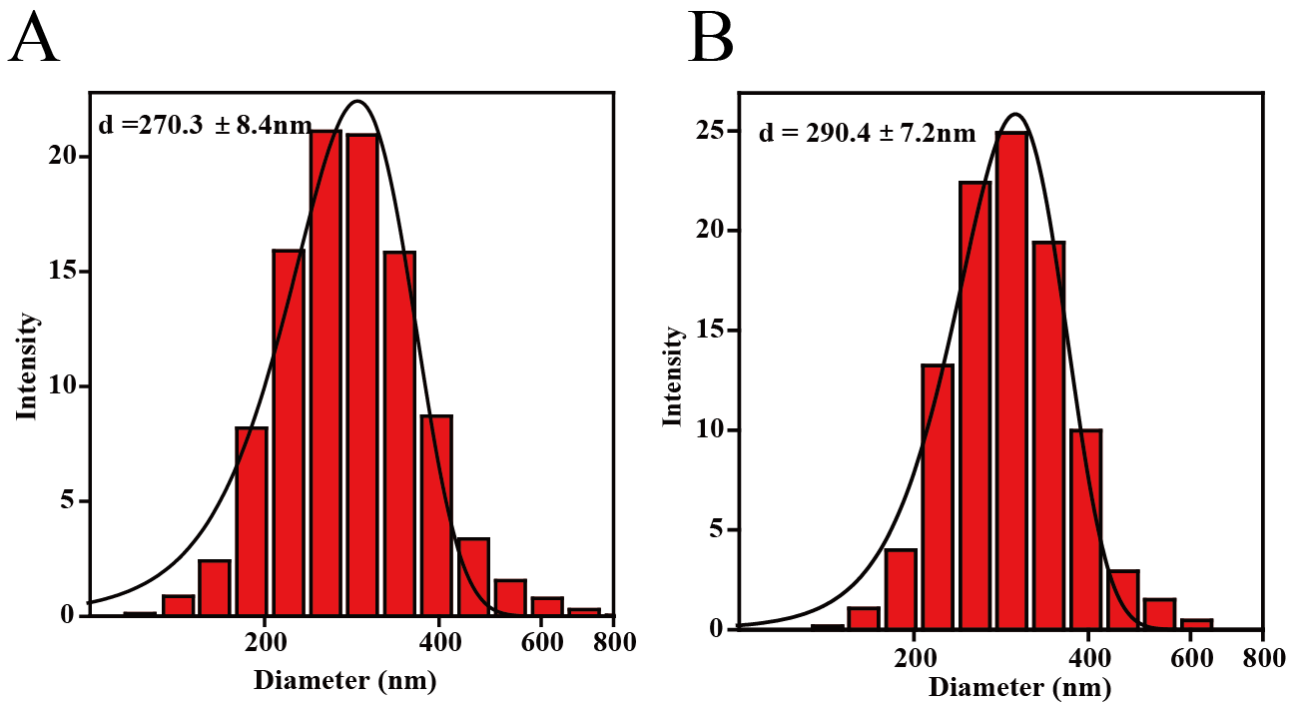

Figure S1. Size distribution of the as-prepared A) PDA nanoparticles and B) the PDA@Van-Ag nanohybrid. 
The UV-Vis spectra (Figure S2A), size (Figure S2B) and zeta potential (Figure S2C) changes of the nanoparticles prepared with increasing $\mathrm{Ag}$ concentrations were recorded. The emergence of new peak $(\sim 500 \mathrm{~nm})$ is ascribed to AgNPs, thus demonstrating the successful deposition of AgNPs on PDA, and the particles increased in size and turned to be less negatively charged with increasing amount of positively charged AgNPs reduced on the surface of PDA, which also evidenced this successful deposition of silver element.

A

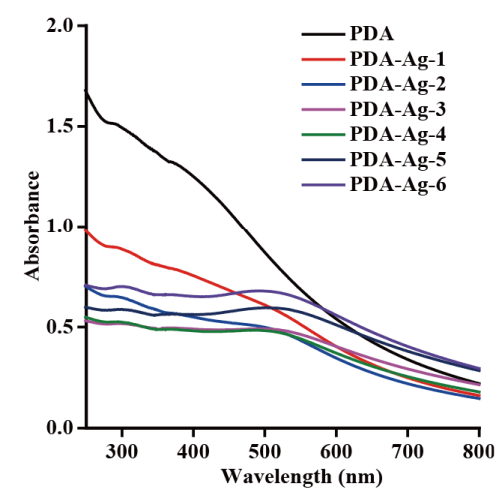

B

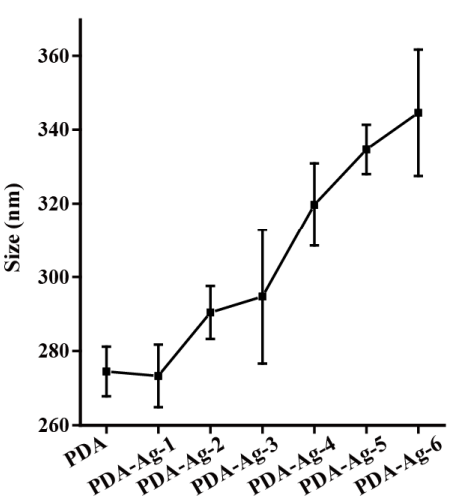

C

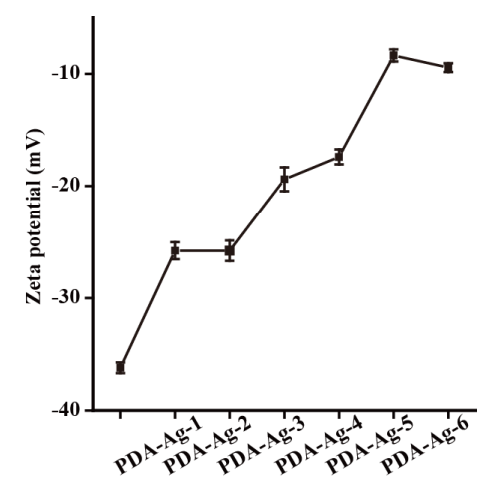

Figure S2. A) UV-Vis spectra B) size and C) zeta potential of PDA modified with increasing amount of silver. 
The polydispersity index (PDI) of different nanoparticles showed that the nanoparticle maintained highly stable with a desirable PDI $(<0.3)$ even with increasing amount of loaded silver (Figure S3).

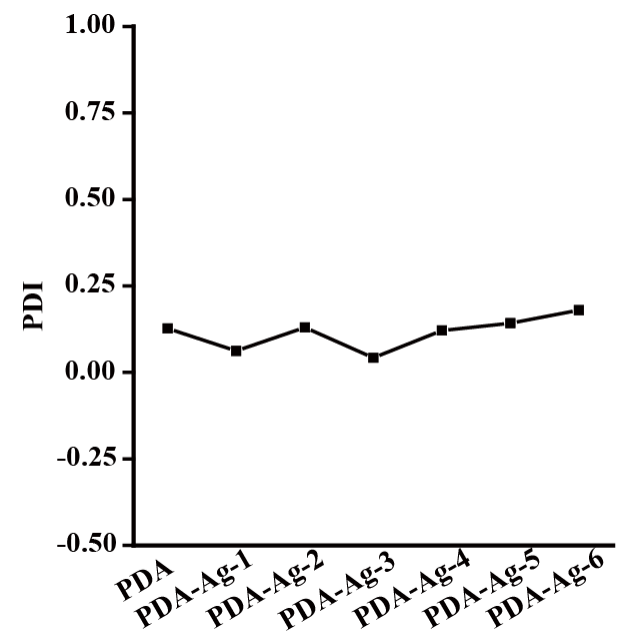

Figure S3. Polydispersity index (PDI) of PDA modified with increasing amount of silver. 


\section{Supporting Information}

The nanohybrid prepared with different concentrations of Ag was characterized by TEM (Figure S4). The corresponding TEM images showed that more AgNPs were deposited on the surface of PDA with increasing concentration of silver precursor while the sphere morphology of PDA was retained.

PDA@Van-Ag-1

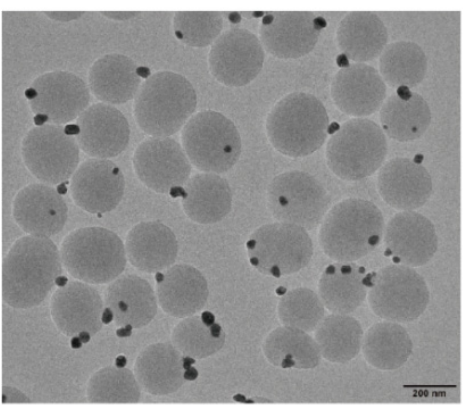

PDA@Van-Ag-4

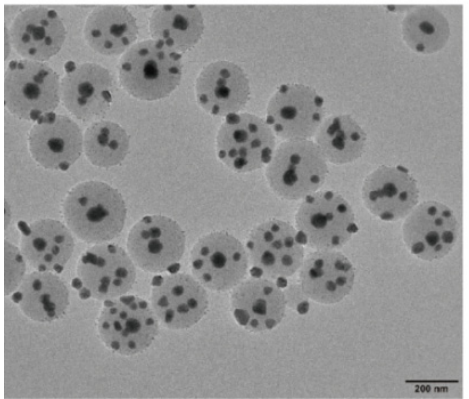

PDA@Van-Ag-2

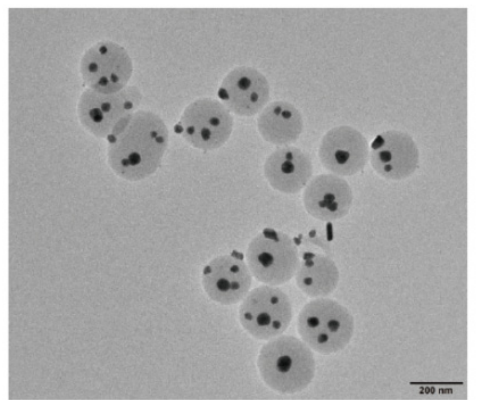

PDA@Van-Ag-5

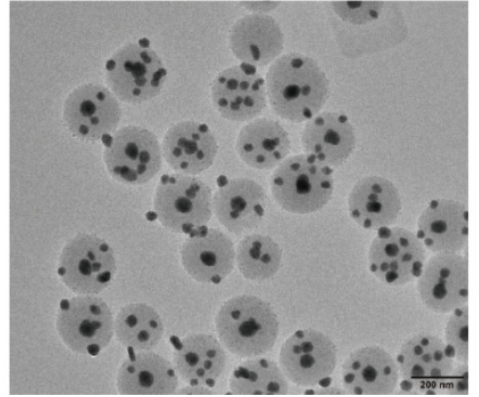

PDA@Van-Ag-3

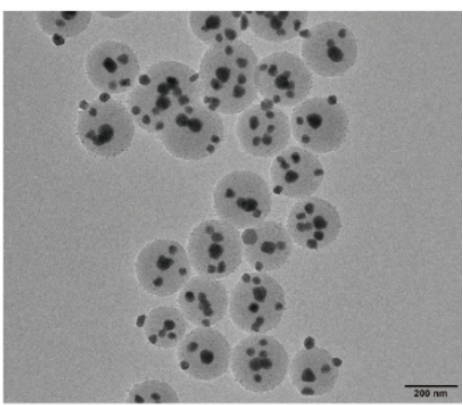

PDA@Van-Ag-6

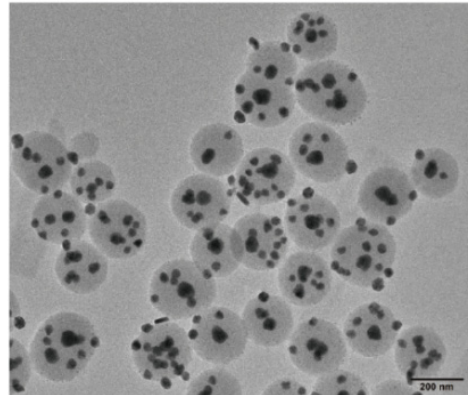

Figure S4. TEM images of PDA@Van-Ag prepared with increasing concentration of silver. Scale bar: $200 \mathrm{~nm}$. 
We first investigated the adsorption ability by mixing PDA with increasing amount of vancomycin as presented in Figure S5A. After centrifugation, the excess vancomycin was removed from the supernatant of PDA/vancomycin mixture and was quantified by UV-Vis spectroscopy. The vancomycin-loaded nanohybrid was also washed several times with water to collect the supernatant for quantifying the weakly adsorbed antibiotics via UV-Vis spectroscopy. Surprisingly, an ultralow desorption of vancomycin from PDA was observed for the subsequent washing steps under a moderate feeding vancomycin $(30 \mu \mathrm{M}$, Figure S5B), indicating a strong PDA-vancomycin interaction. The initial amount of vancomycin was known, thus the PDA-loaded vancomycin was acquired and was increasing with elevated concentration of antibiotics, which verified the strong adsorption of PDA. Based on the strong PDA-vancomycin interaction, the antibiotics-loaded nanohybrid achieved a sustained drug release under a long-term incubation condition (Figure S5C).

A

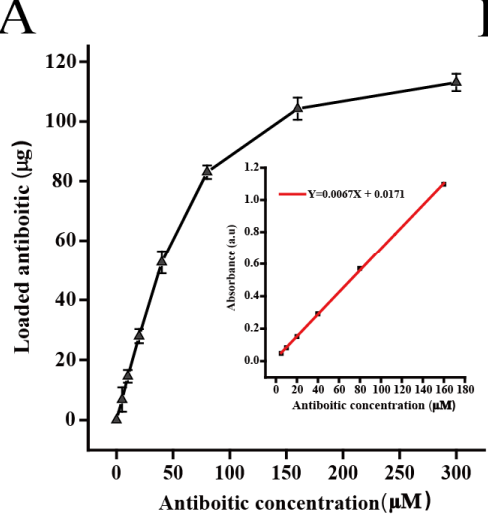

B

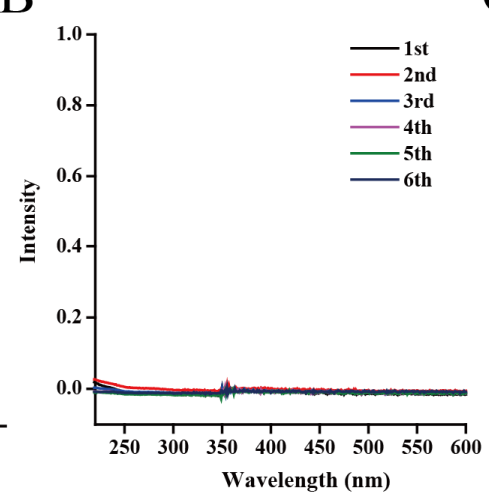

$\mathrm{C}$

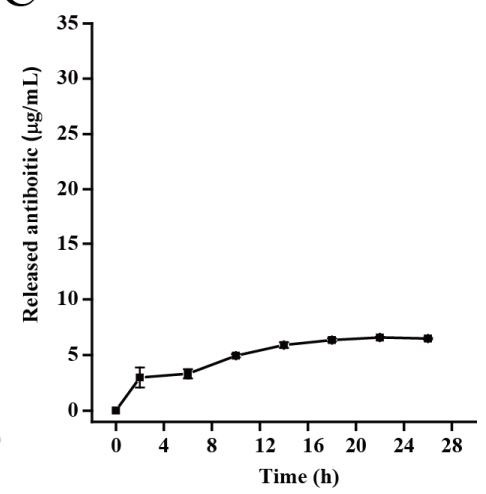

Figure S5. A) Loaded amount of vancomycin onto PDA versus antibiotic concentration. (Insert: the calibration curve of absorbance versus different concentrations of vancomycin.). B) UV-Vis analysis of the supernatant collected from each washing. C) The release profile of vancomycin from this nanohybrid under a long-term incubation condition. 
Due to the excellent photothermal conversion of PDA constitute. The photothermal property of the obtained nanohybrid was studied by exposing to $808 \mathrm{~nm}$ laser irradiation at a fixed power density. PBS was used as the negative control. Obviously, the temperature of PDA@Van-Ag solution increased substantially with prolonged irradiation time and elevated concentration (Figure S6A). However, negligible temperature change was observed in the PBS control group. The thermal images of the PDA@Van-Ag solution with various concentration were recorded by a thermal imaging camera as shown in Figure S6B. The temperature of the nanohybrid elevated with increasing concentrations, which was consistent with the temperature evolution curves. The photothermal stability of the prepared nanohybrid was then evaluated (Figure S6C). During the five repeated laser irradiation cycles, the time-temperature curve was recorded and plotted to investigate the photothermal stability. No variation of the temperature evolution curves was observed, thus demonstrating the remarkably high photothermal stability of the PDA@Van-Ag nanohybrid. In order to investigate the influence of silver on the photothermal effect of the nanoplatform, different PDA nanoparticles with increasing silver amount were irradiated with $808 \mathrm{~nm}$ NIR laser to record their temperature changes. The amount of PDA was set equal in each group. Clearly, the introduction of silver did not compromise their photothermal effects as shown in Figure S6D.
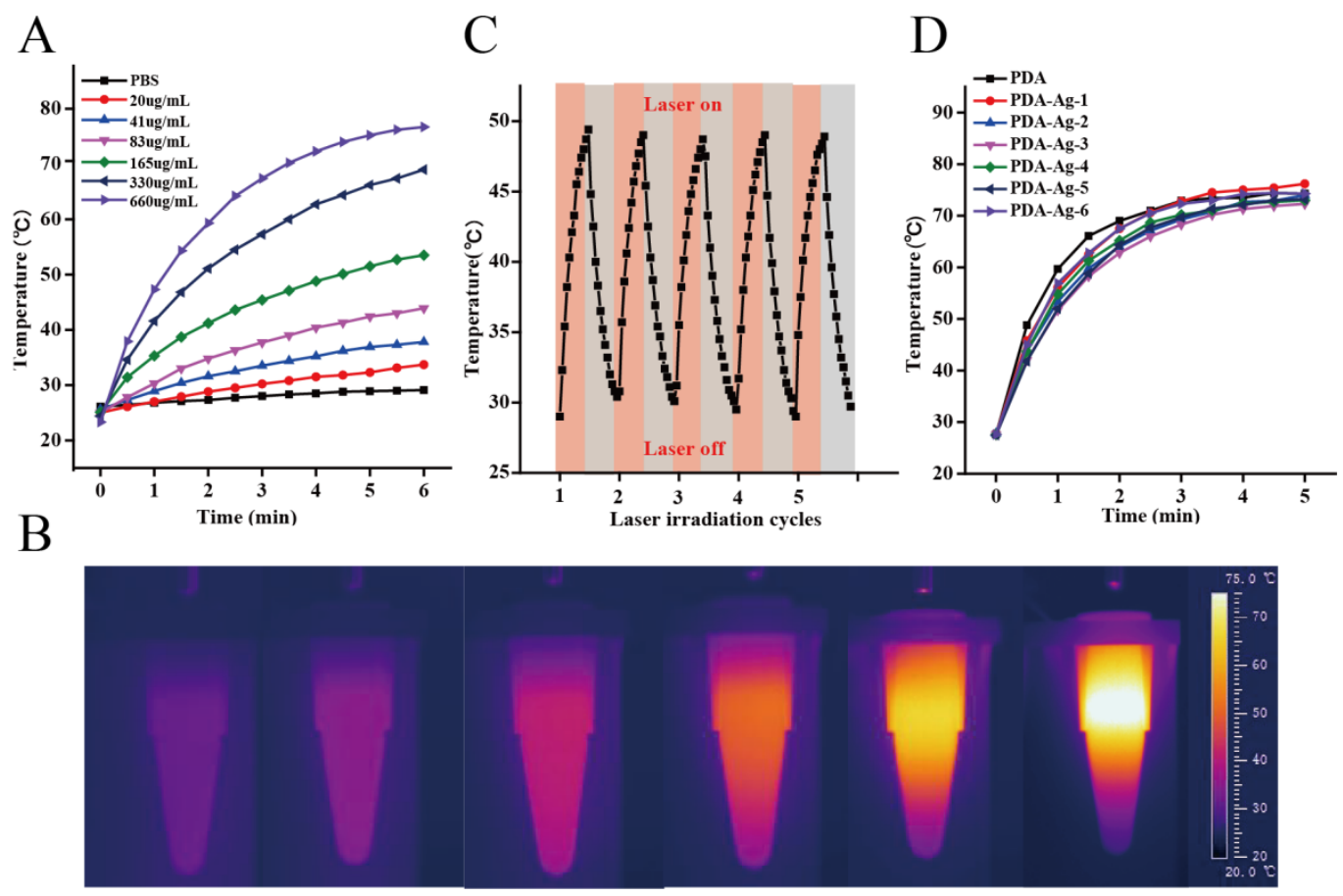

Figure S6. A) Temperature evolution curves and B) their corresponding thermographic pictures of PDA@Van-Ag solutions with increasing concentration under 808nm laser irradiation. C) Photothermal stability of PDA@Van-Ag within 5 laser irradiation cycles. D) Photothermal properties of Ag modified PDA prepared with various concentration of silver solution. The concentration of PDA was set equal in each group (power density of $1 \mathrm{~W} / \mathrm{cm}^{2}$ ). 
In order to evaluate the antibacterial effect of these different nanohybrids under 808 $\mathrm{nm}$ NIR laser irradiation. The S.aureus cells were subjected to different formulations before spreading on the agar plates. After incubation at $37{ }^{\circ} \mathrm{C}$ for $24 \mathrm{~h}$, the formed bacterial colonies were photographed (Figure S7). This hyperthermia-assisted bacteria inactivation could further reduce the required dose of AgNPs to avoid the waste of resources and the potential biotoxicity on humans caused by excessive use.
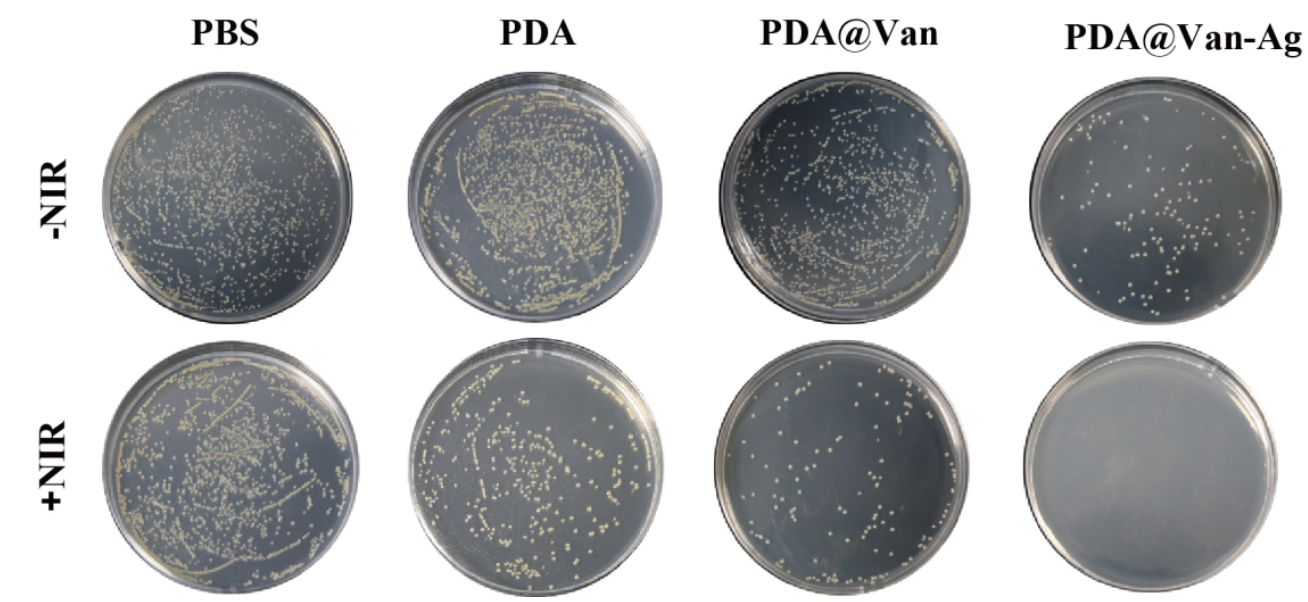

Figure S7. Photographs of agar plates formed by S.aureus cells after exposure to different treatments. 
The typical antibiotic (ciprofloxacin) was selected as a moderate comparison (Figure S8). A high dosage of $8 \mu \mathrm{M}$ ciprofloxacin was needed to eliminate all these S.aureus bacteria, while a lower dose of $4 \mu \mathrm{M}$ is enough to wipe out the majority of E.coli bacteria.

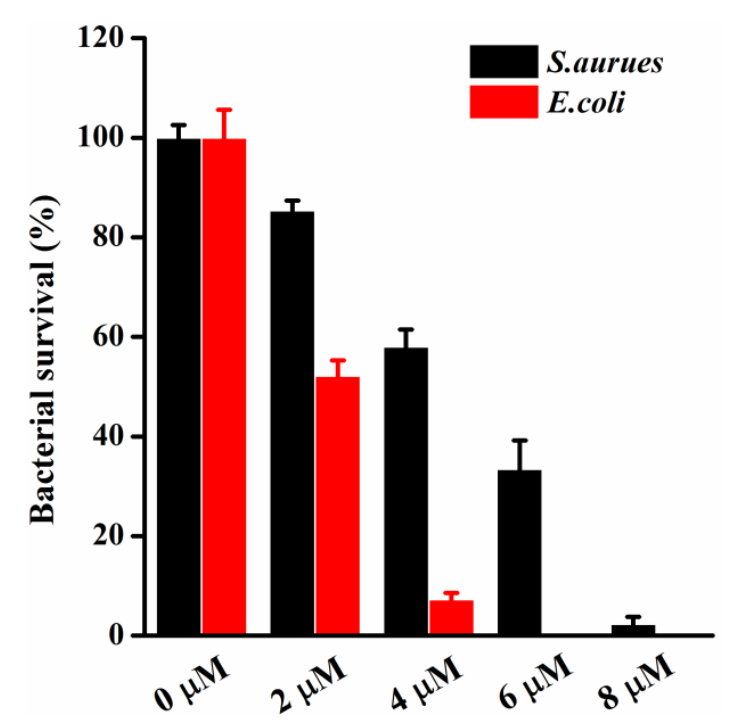

Figure S8. Toxicity of ciprofloxacin with various concentrations against S.aureus and E.coli. 
These differently treated E.coli bacteria were characterized by SEM and confocal fluorescence imaging (Figure S9). Calcein-AM was used to label the viable cells by green fluorescence, while PI with red fluorescence merely penetrated bacteria with damaged cell membranes. As shown in SEM images, the smooth and intact bacterial membrane turned to be collapsed with this PDA@Van-Ag nanohybrid treatment, revealing the outstanding antibacterial performance against E.coli as well. The increased red fluorescence of confocal fluorescence images also evidenced the excellent bacterial membrane damaging ability of this PDA@Van-Ag nanohybrid.
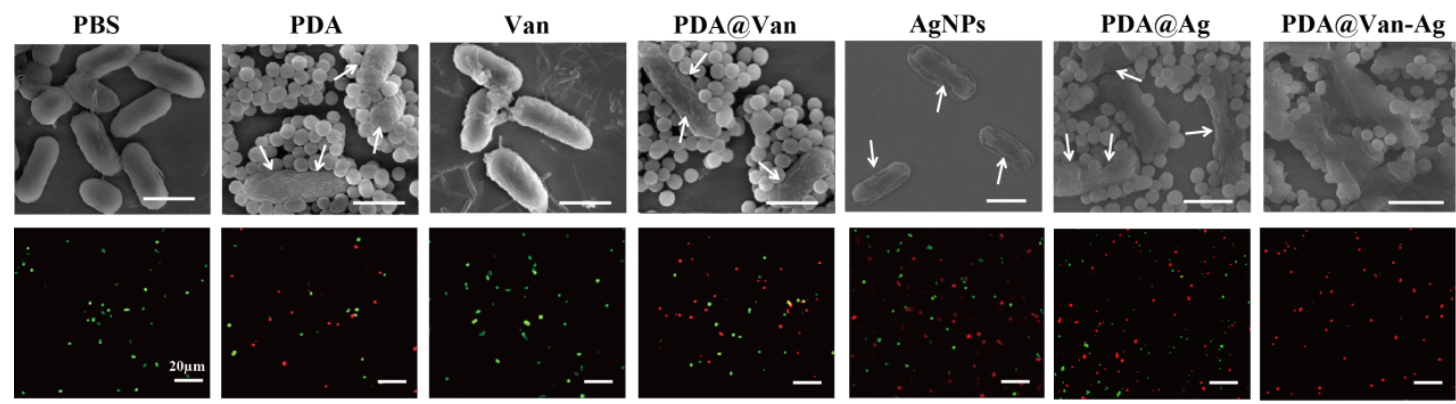

Figure S9. SEM and confocal fluorescence images of E.coli stained with Calcein-AM/PI dyes after different treatments (PBS, PDA, Van, PDA@Van, AgNPs, PDA@Ag andPDA@Van-Ag) 
For in vivo therapeutic application, it is of great importance to demonstrate the low toxicity and systemic safety. The biosafety of PDA@Van-Ag was studied by incubating red blood cells (RBCs) with increasing amount of the nanohybrid. Fresh blood was collected from a healthy female Balb/c mouse. The obtained blood was centrifuged (5000 rpm, $5 \mathrm{~min}$ ) and washed with PBS several times. Subsequently, various concentrations of nanohybrid were incubated with RBCs. Water and PBS were set as the positive control and negative control, respectively. After $1 \mathrm{~h}$ of incubation, these differently treated RBC solutions were subjected to centrifugation (5000 rpm $5 \mathrm{~min}$ ), and the photographs and absorbance of these solutions at $570 \mathrm{~nm}$ were recorded to determine the hemolysis ratio (Figure S10).

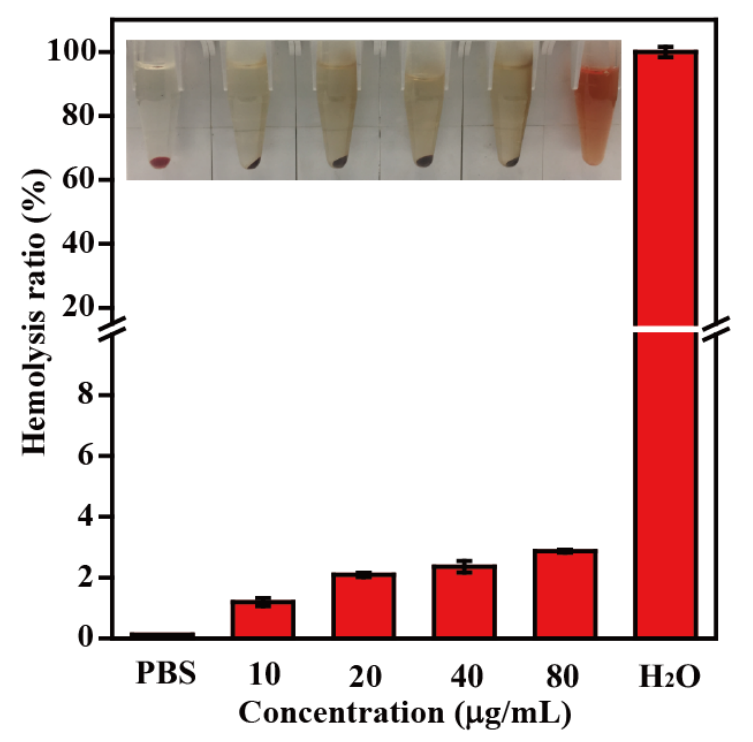

Figure S10. Hemolysis ratio analysis of RBCs after incubation with various concentration of this PDA@Van-Ag nanohybrid. PBS and $\mathrm{H}_{2} \mathrm{O}$ were used as the negative and positive control, respectively. 
MRC-5 cells were used to test the in vitro cytotoxicity of PDA@Van-Ag nanohybrid by a typical methylthiazolyldiphenyl-tetrazolium bromide (MTT) assay (Figure S11). MRC-5 cells were seeded in a 96-well plate with a cell density of $10^{4}$ each well and cultured in Dulbecco's modified Eagle's medium (DMEM) at $37{ }^{\circ} \mathrm{C}$ for $24 \mathrm{~h}$ under $5 \%$ $\mathrm{CO}_{2}$. These nanohybrid solutions $(10,20,40,80 \mu \mathrm{g} / \mathrm{mL})$ were incubated with MRC-5 cells for $24 \mathrm{~h}$ followed by replacement with MTT-dissolved PBS (200 $\mu \mathrm{L}, 0.5$ $\mathrm{mg} / \mathrm{mL}$ ). After $8 \mathrm{~h}$ incubation, the MTT solution was removed and $200 \mu \mathrm{L}$ DMSO was added to each well. After shaking for $20 \mathrm{~min}$, the cell viability was determined through recording the absorbance at $490 \mathrm{~nm}$.

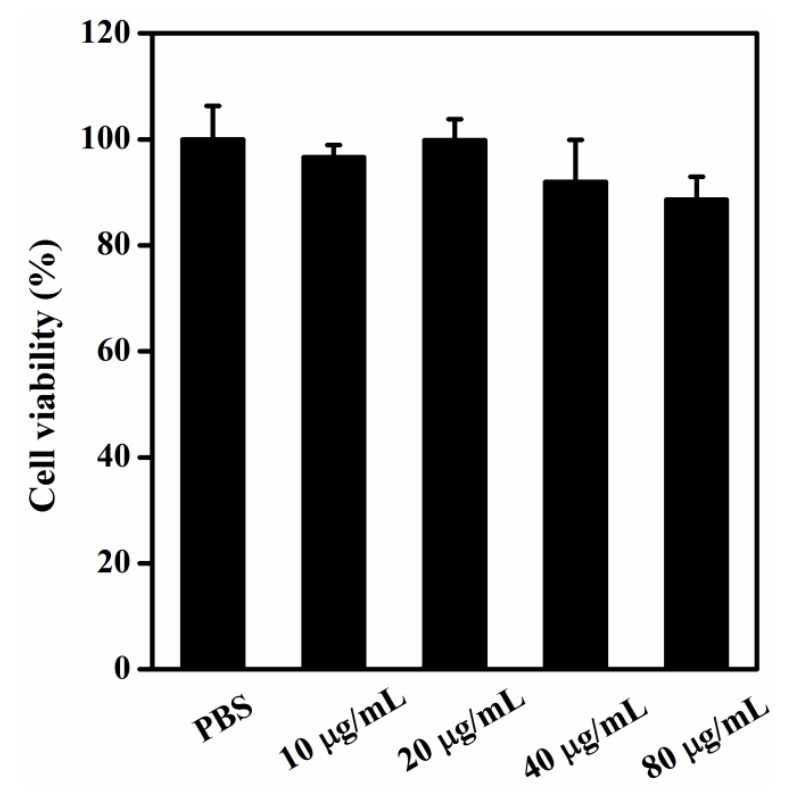

Figure S11. MRC-5 cell viability after incubation with different concentrations of this nanohybrid $(10,20,40,80 \mu \mathrm{g} / \mathrm{mL})$. 
The in vivo toxicity of PDA@Van-Ag was determined by measuring the biodistribution of Ag content in major organs, as acquired by ICP-AES (Table S1). Results demonstrated that the accumulation of Ag was extremely low in the organs of mice that were treated with PDA@Van-Ag nanohybrid, supporting its excellent in vivo biocompatibility.

Table S1. In vivo silver distribution in the main organs (heart, liver, spleen, lung, and kidney) of mice treated with this nanohybrid.

\begin{tabular}{cccccc}
\hline Organs & heart & liver & spleen & lung & kidney \\
\hline $\begin{array}{c}\text { Distribution } \\
\text { amount }\end{array}$ & 0 & $2.87 \times 10^{-3}$ & 0 & 0 & 1.08 \\
$\begin{array}{c}(\% \text { drug } \\
\text { dosage } / g)\end{array}$ & & & & & \\
\hline
\end{tabular}


After the PDA@Van-Ag nanohybrid treatment, the blood of mice was collected for whole blood examination and biochemical analysis in order to further evaluate the in vivo toxicity of this nanoplatform (Figure S12). Main physiological function markers including red blood cells (RBC), white blood cells (WBC), hemoglobin (HGB), mean corpuscular volume (MCV), hematocrit (HCT), red cell distribution width (RDW), mean corpuscular hemoglobin concentration (MCHC), mean corpuscular hemoglobin $(\mathrm{MCH})$ and main liver and kidney function markers involving alanine aminotransferase (ALT), aspartate aminotransferase (AST), creatinine (Cr) and urea nitrogen (UN) were acquired and measured. These parameters were within the normal reference ranges compared with the control group. All these results suggested this nanohybrid showed no toxicity in vivo.
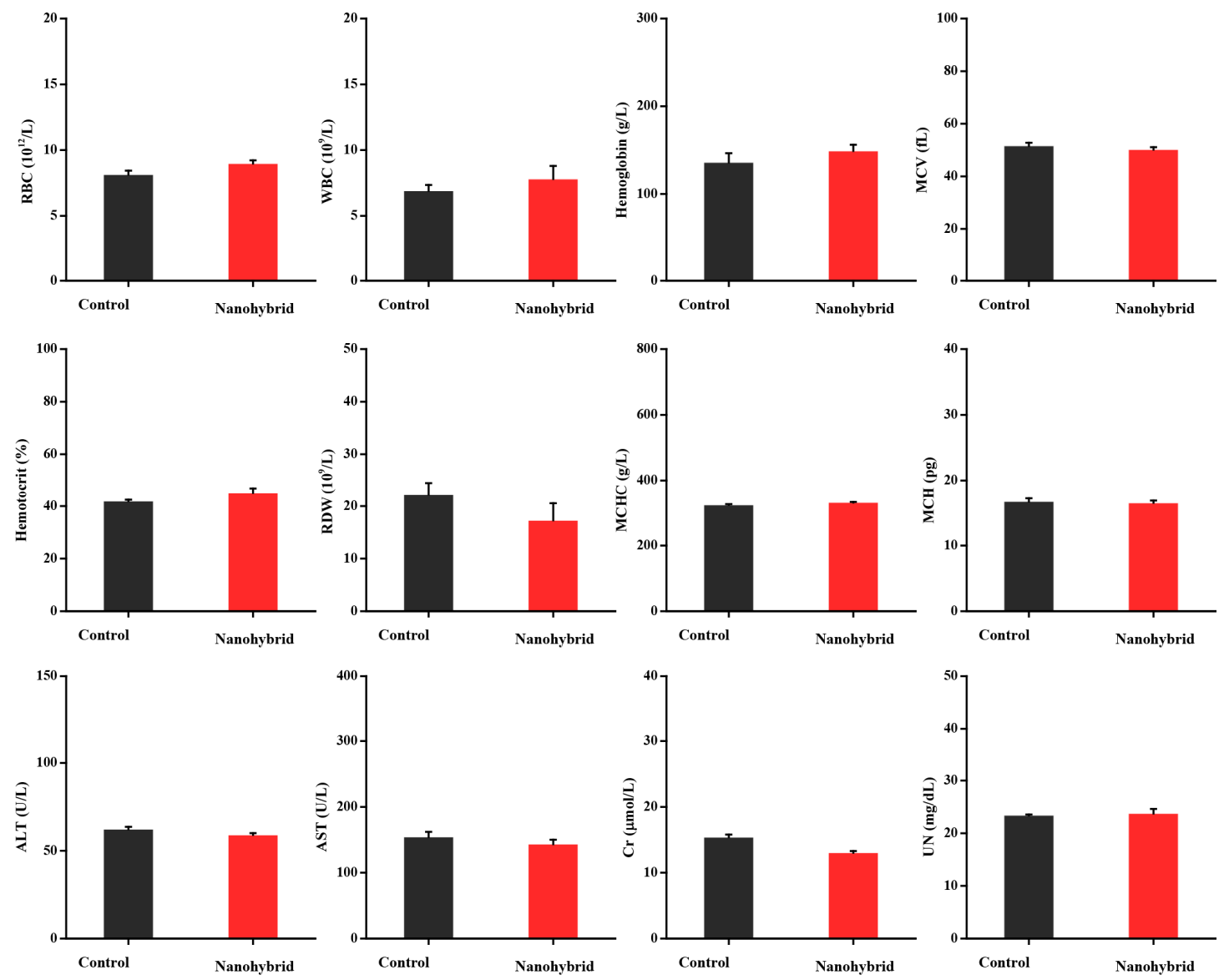

Figure S12. The whole blood testing and biochemical analysis results of mice treated with this PDA@Van-Ag nanohybrid. Here PBS was set as a control. 